\title{
Research Progress of Traditional Chinese Medicine in the Treatment of Carotid Atherosclerosis
}

\author{
Xinya Zhao1, Yongjun Fang2* \\ ${ }^{1}$ The First Clinical Medical College, Shaanxi University of Chinese Medicine, Xianyang 712000, Shaanxi Province, China \\ ${ }^{2}$ The Affiliated Hospital of Shaanxi University of Chinese Medicine, Xianyang 712000, Shaanxi Province, China \\ *Corresponding author: Yongjun Fang, 635340344@qq.com \\ Copyright: (C) 2022 Author(s). This is an open-access article distributed under the terms of the Creative Commons Attribution License (CC \\ BY 4.0), permitting distribution and reproduction in any medium, provided the original work is cited.

\begin{abstract}
Carotid atherosclerosis is a partial manifestation of arteriosclerosis of organism. In recent years, its incidence rate is increasing year by year. Traditional Chinese medicine (TCM) has a broad prospect in the clinical development of CAS treatment by holism concept and the principle of treatment based on syndrome differentiation. This paper reviews the etiology, pathogenesis, TCM dialectics and TCM treatment of carotid atherosclerosis, in order to provide theoretical guidance for TCM treatment of CAS patients.
\end{abstract}

Keywords: Carotid atherosclerosis; CAS; Traditional Chinese medicine; Single Chinese herb

Online publication: January 19, 2022

\section{Introduction}

Atherosclerosis (AS), as a chronic arteritis disease caused by multiple factors, is known as the potential cause of most clinical cardiovascular events ${ }^{[1]}$. And carotid arteriosclerosis is the manifestation of systemic arteriosclerosis in the carotid artery, which gradually worsens with age. In cerebrovascular diseases, $80 \%$ of stroke is ischemic stroke, of which ischemic stroke caused by atherosclerotic carotid stenosis accounts for about $25 \% \sim 30 \%{ }^{[2]}$. CAS has common risk factors and pathological basis with atherosclerosis of coronary artery, cerebral vessels and renal arteries. It is one of the main causes of cardiovascular and cerebrovascular diseases and plays an important role in clinical prevention, diagnosis and treatment ${ }^{[3]}$. When carotid artery stenosis is caused by atherosclerosis, carotid revascularization, including carotid endarterectomy (CEA) and carotid artery stenting (CAS), is the main treatment. However, the operation cost is high, the risk is high, and there are complications such as luxury perfusion, cerebral infarction and restenosis. Long term medication is still needed after the operation. The cognitive function of individual patients will deteriorate temporarily in a short time after operation ${ }^{[4]}$. This paper reviews the etiology, pathogenesis, TCM dialectics and TCM treatment of carotid atherosclerosis, in order to provide theoretical guidance for TCM treatment of CAS patients.

\subsection{Traditional Chinese medicine disease name}

There is no exact disease name in traditional Chinese medicine, and there is no unified understanding of the naming of CAS at present. Most of them are classified and described according to their clinical manifestations and pathological characteristics ${ }^{[5]}$. Traditional Chinese medicine classifies them into "vessel impediment," "palpitation," "chest apoplexy," "stroke” and other categories. 


\subsection{Dialectics of TCM}

Doctors in the past dynasties have different opinions on the etiology and pathology of carotid arteriosclerosis. Their syndrome types are mainly divided into Qi deficiency and blood stasis, phlegm and blood stasis and collaterals stagnation, and excessive fire toxin and heat. Qi deficiency and blood stasis type: Qi is the commander of blood, so the blood can run normally in the pulse, depending on the normal promotion of Qi. If the original Qi is deficient and unable to flow blood, the blood will flow slowly and stay into blood stasis. Clinically, it is common to suffer from Qi deficiency such as fatigue, lack of Qi and lazy speech, accompanied by limb numbness, pain and other blood stasis. The method of supplementing Qi and nourishing blood, as well as promoting blood circulation and removing blood stasis should be used in treatment. Phlegm stasis stagnation type are when the disease lasts for a long time, the Qi and blood decline less, the circulation of Qi and blood is not smooth, the wet stagnation is phlegm, the blood coagulation is blood stasis, the intersection of phlegm and blood stasis is blocked, the coagulation is astringent, the adhesion is difficult to solve, the deficiency is sandwiched in the solid, and the blood vessels in the neck are cemented into blocks over time, which can eventually lead to stroke ${ }^{[6]}$. Fire toxin and heat excess types are such as the evil Qi of fire and heat runs all over the body with Qi and blood, and the pulse is the house of blood. Therefore, the pulse is most vulnerable to hurt by fire and heat, and the pulse channel is damaged, which is difficult to cement for a long time, resulting in hardening and rigidity of the pulse channel. If visible evil such as phlegm and blood stasis stagnate locally, it is the formation of plaque ${ }^{[7]}$. The method of treatment is clearing heat and detoxification, resolving phlegm and removing blood stasis.

\section{Traditional Chinese medicine treatment}

\subsection{Traditional Chinese medicine prescription}

Traditional Chinese medicine has the characteristics of simple medicine, special force, clear intention and benefiting the people. Dialectical treatment can be carried out according to the syndrome types of "Qi deficiency and blood stasis, phlegm and blood stasis and stagnation of collaterals, fire toxin and heat excess." Qi deficiency and blood stasis type: Buyang Huanwu Decoction was used combined with western medicine to treat senile atherosclerotic cerebral infarction ${ }^{[8]}$. Both groups were treated with basic treatment, and the control group was treated with aspirin and clopidogrel. The observation group was treated with Buyang Huanwu Decoction. As a result, the platelet activation ability of the observation group was significantly lower than that of the control group, IMT was significantly lower than that of the control group, and the prognosis was significantly better than that of the control group. Phlegm stasis stagnation type: Danshen Decoction and Erchen decoction was used to treat the syndrome of phlegm and blood stasis in carotid atherosclerosis ${ }^{[9]}$. Both groups were given basic treatment, and the control group took Hedan tablets $(0.32$ $\mathrm{g} /$ tablet) orally, 2 tablets / time, 3 times / d. The observation group was treated with Danshen Decoction and Erchen Decoction. After treatment, the IMT, plaque number, plaque area and plaque thickness in the observation group were lower than those in the control group $(\mathrm{P}<0.01)$, the peak systolic blood flow velocity and end diastolic blood flow velocity were higher than those in the control group $(\mathrm{P}<0.01)$, and the pulsatility index and resistance index were lower than those in the control group $(\mathrm{P}<0.01)$. Fire toxin and heat excess type: Huanglian Jiedu Decoction was used to treat CAS, the control group was treated with conventional western medicine, and the observation group was treated with Huanglian Jiedu Decoction on its basis ${ }^{[10]}$. As a result, the changes of carotid artery diameter, IMT, maximum plaque volume and plaque number in the affected side were significantly reduced, and the observation group was lower than that in the control group.

\subsection{Single traditional Chinese medicine}

The mechanism of single TCM is specific, which can provide reference basis for the compatibility of 
clinical compound and Chinese patent medicine. Astragalus extract was used to treat carotid atherosclerosis in rats, which can reduce the levels of TAG, TC, LDL-C, SDF-1, CXCR4 and VEGF, and then reduce the degree of carotid atherosclerotic plaque in rats ${ }^{[11]}$. Ginkgo biloba extract was used to treat CAS, which can reduce carotid atherosclerotic plaque, reduce inflammatory response, improve blood lipid indicators, and has good safety.

\subsection{Acupuncture and moxibustion of TCM}

Acupuncture and moxibustion are referred to as acupuncture and moxibustion as a whole. They offer the advantages of being simple to use, safe, and have little negative effects. Acupuncture is a technique for regulating the condition of the body by stimulating the human body's meridians and acupuncture sites in order to achieve disease preventative and treatment effects. Moxibustion is the process of aligning moxa at acupuncture sites, lighting it, and relying on the volatilization of moxa leaf oil to reach the depths of the acupoints, adjusting the yin-yang balance of the internal organs. Jun Wang ${ }^{[13]}$ employed acupuncture to treat phlegm dampness-related carotid atherosclerosis. The control group was treated with conventional western medicine, and the observation group was treated with acupuncture on its basis. After treatment, compared with the control group, the plaque area, IMT, serum TNF- $\alpha$, hs-CRP, MMP-9 levels were lower in the observation group, and the incidence of adverse reactions was lower, and the treatment effect was better. Jun Liu ${ }^{[14]}$ and others used moxibustion combined with Banxia Baizhu Tianma Decoction to treat phlegm dampness hypertension with carotid atherosclerosis. The control group was treated with conventional western medicine, and the patients in the treatment group were treated with moxibustion and Banxia Baizhu Tianma Decoction on the basis of their treatment. After treatment, bilateral IMT and plaque area decreased, blood pressure, total cholesterol (TC), triglyceride (TG), low density lipoprotein cholesterol $(\mathrm{LDL}-\mathrm{C})$ and lipoprotein a decreased significantly $(\mathrm{P}<0.05)$, and the treatment group was significantly lower than the control group.

\section{Conclusion}

Atherosclerosis is a condition in which endothelial cell injury and lipid buildup in the artery wall cause atherosclerotic plaque to form over time ${ }^{[15]}$. Many ideas on how it forms exist, including lipid infiltration theory, damage expression theory, hemodynamic theory, inflammatory response theory, and etc. ${ }^{[16]}$. Currently, statins are used to control fat and stable plaque, and aspirin and other medicines are used to inhibit platelet aggregation. Long-term medicine can harm the liver, kidneys, muscles, and coagulation system. Traditional Chinese medicine has demonstrated promising results in the clinical treatment of CAS in recent years, with the benefits of multi-target, multi-channel, and multi-link intervention. However, at present, there is a lack of clinical support from multiple centers and large samples, resulting in changeable treatment guidelines and prescriptions, and it is difficult to clarify the material basis of efficacy. Therefore, the reliability of Chinese medicine in the treatment of CAS still needs further research in order to provide reasonable support for clinical treatment.

\section{Disclosure statement}

The author declares no conflict of interest.

\section{References}

[1] Wang H, Tian Y, 2021, Advancements in the Pathogenesis of Coronary Atherosclerosis in the Adventitia of Blood Vessels. Chinese Journal of Arteriosclerosis, 29(10): 896-902.

[2] Qi B, Jin Q, Hu J, et al., 2021, Research Status and Progress of Neovascularization in Carotid 
Atherosclerotic Plaque. Chinese Journal of Arteriosclerosis, 29(04): 359-362+368.

[3] Guo K, Dong X, Guo R, 2020, Meta Analysis on the Curative Effect of Resolving Phlegm and Promoting Blood Circulation combined with Conventional Western Medicine in the Treatment of Carotid Atherosclerosis. Chinese Journal of Integrative Medicine on Cardio-/Cerebrovascular Disease, 18(17): 2762-2768.

[4] Huo R, Liu Y, Wu H, et al., 2020, Research Progress on the Mechanism of Cognitive Dysfunction in Carotid Artery Stenosis and the Effect of Carotid Revascularization on Cognitive Function. Chinese Journal of Cerebrovascular Disease (Electronic Version), 14(04): 194-198

[5] Li X, Guo Y, 2021, Research Progress in the Treatment of Atherosclerosis with Traditional Chinese Medicine. Henan Medical Research, 30(27): 5180-5183.

[6] Li Y, Gong B, Zhu J, et al., 2021, Chinese Medicine Master Zhu Liangchun's Academic Experience in Treating Carotid Artery Unstable Plaque from Phlegm and Blood Stasis. Chinese Journal of Experimental Traditional Medical Formulae, 1-6. https://doi.org/10.13422/j.cnki.syfjx.20212393

[7] He W, Yang J, Jiang M, et al., 2018, Effect of Heat Clearing and Detoxification Method on HS-CRP and LDL-C in Patients with Carotid Atherosclerosis. Chinese Medicine Modern Distance Education of China, 16(11): 43-45.

[8] Zhu J, 2020, Clinical Study of Buyang Huanwu Decoction Combined with Western Medicine in the Treatment of Senile Atherosclerotic Cerebral Infarction. Chinese Journal of Experimental Traditional Medical Formulae, 41(01): 22-26.

[9] Cao C, Dong X, Huang B, 2021, Protective Effect of Danshen Decoction Combined with Erchen Decoction on Intimal Injury of Carotid Atherosclerosis. Chinese Journal of Experimental Traditional Medical Formulae, 27(07): 86-91.

[10] Liu Y, Sun J, 2019, Observation on Effect of Huanglian Jiedu Decoction on Carotid Atherosclerosis and Its Mechanism. World Chinese Medicine, 14(10): 2688-2692.

[11] Zhou F, Fu P, Zhang X, et al., 2018, Effects of Astragalus Extract on Carotid Atherosclerotic Plaque and Serum SDF-1, CXCR4 and VEGF in Rats. Journal of Emergency in Traditional Chinese Medicine, 27(12): 2115-2119.

[12] Sun K, Yang Q, 2017, Effect of Rosuvastatin Combined with Ginkgo Biloba Extract Tablets on Carotid Atherosclerotic Plaque and Inflammatory Response in Patients with Cerebral Infarction Complicated with Diabetes. Chinese Journal of Gerontology, 37(08): 1933-1935.

[13] Wang J, 2021, Effect Analysis of Acupuncture and Moxibustion on Carotid Atherosclerosis with Phlegm-dampness Constitution. China Health Standard Management, 12(16): 110-113.

[14] Liu J, Li J, 2020, Clinical Observation of Moxibustion Combined with Banxia Baizhu Tianma Decoction in the Treatment of Phlegm-dampness Hypertension with Carotid Atherosclerosis. Guiding Journal of Traditional Chinese Medicine and Pharmacy, 26(01): 69-71 + 78 .

[15] Williams JK, Sukhova GK, Herrington DM, et al., 1998, Pravastatin has Cholesterol-Lowering Independent Effects on the Artery Wall of Atherosclerotic Monkeys. J Am Coll Cardiol, 31(3): 684691.

[16] Li H, Sun S, Song L, 2020, Efficacy and Safety of Heat-clearing and Detoxification in the Treatment of Carotid Atherosclerosis: A Meta-analysis. Chinese Journal of Integrative Medicine on Cardio/Cerebrovascular Disease, 18(14): 2201-2206.

\section{Publisher's note}

Bio-Byword Scientific Publishing remains neutral with regard to jurisdictional claims in published maps and institutional affiliations. 